\title{
Bifurcations and chaos in a three-dimensional generalized Hénon map
}

\author{
Jingjing Zheng ${ }^{1}$, Ziwei Wang ${ }^{2}$, You Li ${ }^{1}$ and Jinliang Wang ${ }^{1 *}$
}

"Correspondence:

jlwang@buaa.edu.cn

'School of Mathematics and

Systems Science, Beihang

University, Beijing, P.R. China

Full list of author information is

available at the end of the article

\section{每 Springer}

\begin{abstract}
This article presents the bifurcation and chaos phenomenon of the three-dimensional generalized Hénon map. We establish the existence and stability conditions for the fixed points of the system. According to the center manifold theorem and bifurcation theory, we get the existence conditions for fold bifurcation, flip bifurcation, and Naimark-Sacker bifurcation of the system. Finally, the bifurcation diagrams, Lyapunov exponents, phase portraits are carried out to illustrate these theoretical results. Furthermore, as parameter varies, new interesting dynamics behaviors, including from stable fixed point to attracting invariant cycle and to chaos, from periodic-10 to chaos, etc., are observed from the numerical simulations. In particular, we find the double-cycle phenomenon from bifurcation diagrams and phase portraits.
\end{abstract}

MSC: 34C23; 39A28; 39A33

Keywords: Hénon map; Fold bifurcation; Flip bifurcation; Naimark-Sacker bifurcation; Double-cycle

\section{Introduction}

Classic Hénon map is a two-dimensional discrete time system. It was first defined in [1] via constant Jacobian determinant. Since the late 1970s, the Hénon map has attracted considerable attention of investigators and has been widely studied. The Hénon map has a wide range of applications in many areas, there are more and more researchers [2-5] to study the Hénon map. Besides, many sophisticated algorithms have been developed on the basis of the Hénon map recently. In paper [6], the Hénon map is used in image encryption, and its encryption speed is faster than other image encryption schemes. Alligood and Sauer [7] explored the specific properties of periodic orbits of the Hénon map. Compound windows of the Hénon map were introduced by Lorenz [8]. The authors in paper [9] mainly studied the application of the fractional order derived in the generalized hyperchaotic Hénon map. In addition, researchers also have proposed different types of the generalized Hénon $[10,11]$ map, which exhibit more complex dynamics.

In 1900, Baier and Klein [10] defined the $n$-dimensional generalized Hénon map. And then Richter [12] collected analytical and numerical results for maps of lower and higher dimensions of the $n$-dimensional generalized Hénon map in 2002. Since the threedimensional Hénon map [13-15] is of great importance in the study of high-dimensional Hénon map, more and more investigators consider the three-dimensional Hénon map. For example, the authors in [16] proposed a new interesting three-dimensional generalized

(c) The Author(s) 2018. This article is distributed under the terms of the Creative Commons Attribution 4.0 International License (http://creativecommons.org/licenses/by/4.0/), which permits unrestricted use, distribution, and reproduction in any medium, provided you give appropriate credit to the original author(s) and the source, provide a link to the Creative Commons license, and indicate if changes were made. 
Hénon map. Different types of Lorenz-type attractors in the three-dimensional Hénon map are presented in [17]. Gonchenko [18] investigated the pseudo-hyperbolic attractors in the three-dimensional generalized Hénon map.

However, the study of bifurcations and chaos for the three-dimensional generalized Hénon map is still rare. According to [12], we consider the $m$-dimensional generalized Hénon map with the following form:

$$
\left\{\begin{array}{l}
x_{1}(n+1)=a-x_{m-1}^{2}(n)+b x_{m}(n), \quad j=1, \ldots, m-1 . \\
x_{j+1}(n+1)=x_{j}(n),
\end{array}\right.
$$

In particular, we consider the case of $m=3$. Let $x_{1}=x, x_{2}=y, x_{3}=z$, map (1) can be rewritten as

$$
\left\{\begin{array}{l}
x_{n+1}=a-y_{n}^{2}+b z_{n} \\
y_{n+1}=x_{n} \\
z_{n+1}=y_{n}
\end{array}\right.
$$

This paper aims at exploring the dynamical behaviors of system (2).

We use the center manifold theorem and bifurcation theory [19] to explore the fold bifurcation, flip bifurcation, and Naimark-Sacker bifurcation of system (2). Then we illustrate our theoretical results by numerical simulations. From the numerical simulation results, we observe many new interesting dynamical behaviors, such as bifurcation, periodic orbit, invariant cycle, chaos, and periodic window. In particular, bifurcation diagrams and phase portraits show coexistence of unstable invariant cycle and stable invariant cycle in system (2) for $b=-0.85$.

The main content of the paper is as follows. In Sect. 2, we investigate the existence and stability of the fixed points of system (2). In Sect. 3, the analytical conditions of codimension one bifurcations including fold bifurcation, flip bifurcation, and Naimark-Sacker bifurcation are derived on the basis of center manifold theorem and bifurcation theory. In Sect. 4, we give the numerical simulations by using MATLAB to illustrate our theoretical results. From those numerical results we find that system (2) has new interesting complex dynamical behaviors. Finally, we give a brief conclusion of the paper in Sect. 5 .

\section{Existence and stability of fixed points}

Direct computations from system (2) show that the fixed point $Z^{*}\left(x^{*}, y^{*}, z^{*}\right)$ satisfies

$$
\left\{\begin{array}{l}
x^{*}=a-y^{* 2}+b z^{*} \\
y^{*}=x^{*} \\
z^{*}=y^{*}
\end{array}\right.
$$

We can get the relationship between three elements of the fixed point $Z^{*}$, that is, $x^{*}=y^{*}=$ $z^{*}$, besides $x^{*}$ satisfies $x^{* 2}-(b-1) x^{*}-a=0$.

Then we obtain the proposition for the existence of the fixed points.

\section{Proposition 1}

(1) If $a>a_{0}$, system (2) has two fixed points $Z_{1}^{*}\left(x_{1}^{*}, y_{1}^{*}, z_{1}^{*}\right)$ and $Z_{2}^{*}\left(x_{2}^{*}, y_{2}^{*}, z_{2}^{*}\right)$;

(2) If $a=a_{0}$, system (2) has a unique fixed point $Z_{0}^{*}\left(x_{0}^{*}, y_{0}^{*}, z_{0}^{*}\right)$; 
(3) If $a<a_{0}$, system (2) has no fixed point,

where $a_{0}=-\frac{(b-1)^{2}}{4}, x_{i}^{*}=y_{i}^{*}=z_{i}^{*}, i=0,1,2, x_{0}^{*}=\frac{b-1}{2}, x_{1}^{*}=\frac{1}{2}\left[(b-1)-\sqrt{4 a+(b-1)^{2}}\right], x_{2}^{*}=$ $\frac{1}{2}\left[(b-1)+\sqrt{4 a+(b-1)^{2}}\right]$.

Next, we give the condition for the stability of the fixed points $Z_{i}^{*}(i=1,2)$ of system (2). The Jacobian matrix $J$ of system (2) associated with the fixed point $Z_{i}^{*}$ is

$$
J=\left(\begin{array}{ccc}
0 & -2 y_{i}^{*} & b \\
1 & 0 & 0 \\
0 & 1 & 0
\end{array}\right)
$$

The characteristic equation of $J$ is

$$
\lambda^{3}+2 y_{i}^{*} \lambda-b=0
$$

Let $\lambda=\frac{\mu+1}{\mu-1},(4)$ becomes

$$
\mu^{3}+a_{1} \mu^{2}+a_{2} \mu+a_{3}=0
$$

where $a_{1}=\frac{3+3 b-2 y_{i}^{*}}{1-b+2 y_{i}^{*}}, a_{2}=\frac{3-3 b-2 y_{i}^{*}}{1-b+2 y_{i}^{*}}, a_{3}=\frac{1+b+2 y_{i}^{*}}{1-b+2 y_{i}^{*}}$.

Obviously, $|\lambda|<1$ is equivalent to $\operatorname{Re}[\mu]<0$. So, the fixed point $Z_{i}^{*}$ of system (2) is stable when $\operatorname{Re}[\mu]<0$.

By Routh-Hurwitz theorem, we know that $\operatorname{Re}[\mu]<0$ if and only if the following conditions hold: $a_{i}>0, i=1,2,3, D_{1}=a_{1}>0, D_{2}=a_{1} a_{2}-a_{3}>0$, and $D_{3}=a_{3} D_{2}>0$. That is,

$$
\left\{\begin{array}{l}
a_{1}>0, \\
a_{3}>0, \\
a_{1} a_{2}-a_{3}>0 .
\end{array}\right.
$$

By analyzing the above conditions, we find that the fixed point $Z_{1}^{*}$ is always unstable, and the fixed point $Z_{2}^{*}$ is stable if

$$
\left\{\begin{array} { l } 
{ 0 \leq b < 1 , } \\
{ - \frac { ( b - 1 ) ^ { 2 } } { 4 } < a < \frac { ( b - 1 ) ^ { 2 } ( b + 1 ) ( b + 3 ) } { 4 } }
\end{array} \text { or } \quad \left\{\begin{array}{l}
-1<b<0, \\
b^{2}-\frac{(b-1)^{2}}{4}<a<\frac{(b-1)^{2}(b+1)(b+3)}{4} .
\end{array}\right.\right.
$$

We summarize the above results about the stability of the fixed points as follows.

\section{Proposition 2}

(1) The fixed point $Z_{1}^{*}$ of (2) is always unstable;

(2) The fixed point $Z_{2}^{*}$ of (2) is stable if one of the following conditions is satisfied:

(i) $b \in[0,1)$ and $a \in\left(a_{0}, a_{h}\right)$,

(ii) $b \in(-1,0)$ and $a \in\left(a^{*}, a_{h}\right)$,

where $a_{0}=-\frac{(b-1)^{2}}{4}, a^{*}=b^{2}-\frac{(b-1)^{2}}{4}, a_{h}=\frac{(b-1)^{2}(b+1)(b+3)}{4}$.

The stable region of $Z_{2}^{*}$ of (2) in the parametric space is shown in Fig. 1 . In the next section, we will prove that the boundary of the stable region of $Z_{2}^{*}$ consists of codimension one bifurcations. 
Figure 1 The stable region and its boundary curves, HB, fold, and flip of $Z_{2}^{*}$ in the $(b, a)$-plane, where $H B$, fold, and flip represent Naimark-Sacker, fold, and flip bifurcation curves, respectively

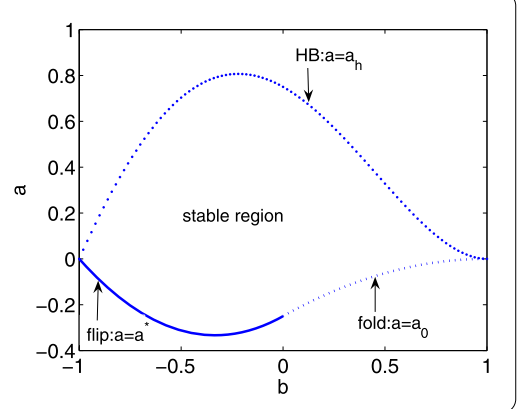

\section{Codimension one bifurcations}

The sufficient conditions for the existence of fold bifurcation, flip bifurcation, and Naimark-Sacker bifurcation are deduced in this section. In order to get the conditions for the existence of the bifurcations, the parameter $a$ is taken as the bifurcation parameter. Since the fixed point $Z_{1}^{*}$ is always unstable, we just analyze the bifurcations of the fixed point $Z_{2}^{*}$ in this section.

The characteristic equation of $J$ associated with $Z_{2}^{*}$ is

$$
\lambda^{3}+\left[(b-1)+\sqrt{4 a+(b-1)^{2}}\right] \lambda-b=0 .
$$

\subsection{Fold bifurcation}

If $a=a_{0}$ and $b \in(0,1)$, the system of (2) has a unique fixed point $Z_{2}^{*}=Z_{0}^{*}$. The roots of (6) are $\lambda_{1}\left(a_{0}\right)=1, \lambda_{2,3}\left(a_{0}\right)=\frac{-1 \pm \sqrt{1-4 b}}{2}$. Particularly, if $b \in\left(0, \frac{1}{4}\right), \lambda_{2,3}\left(a_{0}\right)=\frac{-1 \pm \sqrt{1-4 b}}{2}$ are a pair of real roots; if $b=\frac{1}{4}, \lambda_{2,3}\left(a_{0}\right)=-\frac{1}{2}$; if $b \in\left(\frac{1}{4}, 1\right), \lambda_{2,3}\left(a_{0}\right)=\frac{-1 \pm i \sqrt{4 b-1}}{2}$ are a pair of conjugate complex roots. So we analyze the fold bifurcation in three cases, i.e., Case (I) $b \in\left(0, \frac{1}{4}\right)$, Case (II) $b=\frac{1}{4}$, and Case (III) $b \in\left(\frac{1}{4}, 1\right)$. For the fold bifurcation, it requires $\left|\lambda_{2,3}\left(a_{0}\right)\right| \neq 1$. In fact, it is not hard to prove $\left|\lambda_{2,3}\left(a_{0}\right)\right|<1$ as $b \in(0,1)$.

Let $\bar{x}=x-x_{0}^{*}, \bar{y}=y-y_{0}^{*}, \bar{z}=z-z_{0}^{*}$, and $\bar{a}=a-a_{0}$, system (2) becomes

$$
\left(\begin{array}{c}
\bar{x} \\
\bar{y} \\
\bar{z} \\
\bar{a}
\end{array}\right) \longmapsto\left(\begin{array}{cccc}
0 & 1-b & b & 1 \\
1 & 0 & 0 & 0 \\
0 & 1 & 0 & 0 \\
0 & 0 & 0 & 1
\end{array}\right)\left(\begin{array}{c}
\bar{x} \\
\bar{y} \\
\bar{z} \\
\bar{a}
\end{array}\right)+\left(\begin{array}{c}
-\bar{y}^{2} \\
0 \\
0 \\
0
\end{array}\right)
$$

For Case (I), let $(\bar{x}, \bar{y}, \bar{z}, \bar{a})^{\prime}=T(u, v, w, \mu)^{\prime}$, then map (7) becomes

$$
\left(\begin{array}{c}
u \\
v \\
w \\
\mu
\end{array}\right) \longmapsto\left(\begin{array}{cccc}
1 & 1 & 0 & 0 \\
0 & 1 & 0 & 0 \\
0 & 0 & -\frac{1+\sqrt{1-4 b}}{2} & 0 \\
0 & 0 & 0 & \frac{-1+\sqrt{1-4 b}}{2}
\end{array}\right)\left(\begin{array}{c}
u \\
v \\
w \\
\mu
\end{array}\right)+f(u, v, w, \mu)\left(\begin{array}{c}
2 \sqrt{1-4 b} \\
0 \\
3-\sqrt{1-4 b} \\
-3-\sqrt{1-4 b}
\end{array}\right)
$$

where

$$
T=\left(\begin{array}{cccc}
1 & 2 & \left(\frac{1+\sqrt{1-4 b}}{2}\right)^{2} & \left(\frac{-1+\sqrt{1-4 b}}{2}\right)^{2} \\
1 & 1 & -\frac{1+\sqrt{1-4 b}}{2} & \frac{-1+\sqrt{1-4 b}}{2} \\
1 & 0 & 1 & 1 \\
0 & 2+b & 0 & 0
\end{array}\right)
$$


and

$$
f(u, v, w, \mu)=-\frac{\left(u+v+\frac{-1-\sqrt{1-4 b}}{2} w+\frac{-1+\sqrt{1-4 b}}{2} \mu\right)^{2}}{2(2+b) \sqrt{1-4 b}} .
$$

The center manifold theory [19] tells us that, in order to study the stability and the fold bifurcation of $(\bar{x}, \bar{y}, \bar{z})=(0,0,0)$ near $\bar{a}=0$, we only need to consider a one-parameter family of maps which is the reduced form of (7) on a center manifold $W^{c}(0)$, where $W^{c}(0)=$ $\left\{(u, v, w, \mu) \mid w=h_{1}(u, v), \mu=h_{2}(u, v), h_{i}(0,0)=0, D h_{i}(0,0)=0, i=1,2\right\}$. So, we assume that $h_{i}(u, v)=\alpha_{i} u^{2}+\beta_{i} u v+\gamma_{i} v^{2}+O(3), i=1,2$, where $O(3)$ is the sum of all terms whose degree is greater than 2 .

From the existence theorem for center manifolds, the restrictions of map (8) can be obtained as follows:

$$
u \longmapsto F(u, v),
$$

where $F(u, v)=u+v+2 \sqrt{1-4 b} f\left(u, v, h_{1}(u, v), h_{2}(u, v)\right)=u+v-\frac{(u+v)^{2}}{2+b}+O(3)$. Since the fold bifurcation is independent of the term $O(3)$ of $F(u, v)$, there is no need to compute $h_{i}(u, v)$, $i=1$, 2. Since $F(0,0)=0, F_{u}(0,0)=1, F_{v}(0,0)=1, F_{u u}(0,0)=-\frac{2}{b+2}<0$. So, the fixed point $(u, v)=(0,0)$ is a fold bifurcation point for map (9).

As for Cases (II) and (III), we are still able to achieve the same results using only similar analysis and transformation, $(\bar{x}, \bar{y}, \bar{z}, \bar{a})^{\prime}=T_{i}(u, v, w, \mu)^{\prime}, i=1,2$, respectively, where

$$
\begin{aligned}
T_{1} & =\left(\begin{array}{cccc}
1 & 2 & 1 / 4 & -1 \\
1 & 1 & -1 / 2 & 1 \\
1 & 0 & 1 & 0 \\
0 & 9 / 4 & 0 & 0
\end{array}\right), \\
T_{2} & =\left(\begin{array}{cccc}
1 & 2 & -\sqrt{b-1 / 4} & 1 / 2-b \\
1 & 1 & \sqrt{b-1 / 4} & -1 / 2 \\
1 & 0 & 0 & 1 \\
0 & 2+b & 0 & 0
\end{array}\right) .
\end{aligned}
$$

Summarizing the above serious analysis, we can get the following theorem.

Theorem 3 Map (2) undergoes a fold bifurcation at $Z_{0}^{*}$ if the following conditions are satisfied: $b \in(0,1)$ and $a=a_{0}$. Moreover, the two fixed points, $Z_{1}^{*}$ and $Z_{2}^{*}$, bifurcate from $Z_{0}^{*}$ for $a>a_{0}$, coalesce as the fixed point $Z_{0}^{*}$ at $a=a_{0}$, and disappear for $a<a_{0}$.

\subsection{Flip bifurcation}

The conditions for the flip bifurcation occurring at the fixed point $Z_{2}^{*}$ of map (2) are elaborated in this subsection. If $a=a^{*}$ and $b \in(-1,0), x_{2}^{*}=y_{2}^{*}=z_{2}^{*}=-\frac{b+1}{2}$. The roots of (6) are $\lambda_{1}\left(a^{*}\right)=-1, \lambda_{2,3}\left(a^{*}\right)=\frac{1 \pm \sqrt{1+4 b}}{2}$. Particularly, if $b \in\left(-\frac{1}{4}, 0\right), \lambda_{2,3}\left(a^{*}\right)=\frac{1 \pm \sqrt{1+4 b}}{2}$; if $b=-\frac{1}{4}$, $\lambda_{2,3}\left(a^{*}\right)=\frac{1}{2}$; if $b \in\left(-1,-\frac{1}{4}\right), \lambda_{2,3}\left(a^{*}\right)=\frac{1 \pm i \sqrt{-(1+4 b)}}{2}$. So we analyze the flip bifurcation in three cases: $b \in\left(-\frac{1}{4}, 0\right), b=-\frac{1}{4}$, and $b \in\left(-1,-\frac{1}{4}\right)$. For the flip bifurcation, it requires that $\left|\lambda_{2,3}\left(a^{*}\right)\right| \neq 1$. In fact, it is easy to prove that $\left|\lambda_{2,3}\left(a^{*}\right)\right|<1$ as $b \in(-1,0)$. 
Let $\bar{x}=x-x_{2}^{*}, \bar{y}=y-y_{2}^{*}, \bar{z}=z-z_{2}^{*}, \bar{a}=a-a^{*}$, then system (2) becomes

$$
\left(\begin{array}{c}
\bar{x} \\
\bar{y} \\
\bar{z} \\
\bar{a}
\end{array}\right) \mapsto\left(\begin{array}{cccc}
0 & 1+b & b & 1 \\
1 & 0 & 0 & 0 \\
0 & 1 & 0 & 0 \\
0 & 0 & 0 & 1
\end{array}\right)\left(\begin{array}{c}
\bar{x} \\
\bar{y} \\
\bar{z} \\
\bar{a}
\end{array}\right)+\left(\begin{array}{c}
-\bar{y}^{2} \\
0 \\
0 \\
0
\end{array}\right) .
$$

For the case of $b \in\left(-\frac{1}{4}, 0\right)$, let $(\bar{x}, \bar{y}, \bar{z}, \bar{a})^{\prime}=\hat{T}(u, v, w, \mu)^{\prime}$, then map (10) becomes

$$
\begin{aligned}
\left(\begin{array}{c}
u \\
v \\
w \\
\mu
\end{array}\right) \longmapsto & \left(\begin{array}{cccc}
-1 & 0 & 0 & 0 \\
0 & 1 & 0 & 0 \\
0 & 0 & \frac{1-\sqrt{1+4 b}}{2} & 0 \\
0 & 0 & 0 & \frac{1+\sqrt{1+4 b}}{2}
\end{array}\right)\left(\begin{array}{c}
u \\
v \\
w \\
\mu
\end{array}\right) \\
& +\hat{f}(u, v, w, \mu)\left(\begin{array}{c}
2 \sqrt{1+4 b} \\
0 \\
-3-\sqrt{1+4 b} \\
3-\sqrt{1+4 b}
\end{array}\right),
\end{aligned}
$$

where

$$
\hat{T}=\left(\begin{array}{cccc}
1 & -\frac{1}{2 b} & \left(\frac{1-\sqrt{1+4 b}}{2}\right)^{2} & \left(\frac{1+\sqrt{1+4 b}}{2}\right)^{2} \\
-1 & -\frac{1}{2 b} & \frac{1-\sqrt{1+4 b}}{2} & \frac{1+\sqrt{1+4 b}}{2} \\
1 & -\frac{1}{2 b} & 1 & 1 \\
0 & 1 & 0 & 0
\end{array}\right)
$$

and

$$
\hat{f}(u, v, w, \mu)=-\frac{\left(-u-\frac{1}{2 b} v+\frac{1-\sqrt{1+4 b}}{2} w+\frac{1+\sqrt{1+4 b}}{2} \mu\right)^{2}}{2(2-b) \sqrt{1+4 b}} .
$$

From the center manifold theory [19], we can get the conditions of the stability and the flip bifurcation of $(\bar{x}, \bar{y}, \bar{z})=(0,0,0)$ near $\bar{a}=0$ by investigating a one-parameter family of maps which is the reduced form of (10) on a center manifold $W^{c}(0)$, where $W^{c}(0)=$ $\left\{(u, v, w, \mu) \mid w=\hat{h}_{1}(u, v), \mu=\hat{h}_{2}(u, v), \hat{h}_{i}(0,0)=0, D \hat{h}_{i}(0,0)=0, i=1,2\right\}$. So, we assume that $\hat{h}_{i}(u, v)=\hat{\alpha}_{i} u^{2}+\hat{\beta}_{i} u v+\hat{\gamma}_{i} v^{2}+O(3), i=1,2$.

Because of invariance of the center manifold [19], we have that

$$
\left\{\begin{array}{l}
\hat{h}_{1}\left(-u+2 \sqrt{1+4 b} \hat{f}\left(u, v, \hat{h}_{1}(u, v), \hat{h}_{2}(u, v)\right), v\right) \\
\left.\quad=\frac{1-\sqrt{1+4 b}}{2} \hat{h}_{1}(u, v)-(3+\sqrt{1+4 b}) \hat{f}\left(u, v, \hat{h}_{1}(u, v), \hat{h}_{2}(u, v)\right)\right), \\
\hat{h}_{2}\left(-u+2 \sqrt{1+4 b} \hat{f}\left(u, v, \hat{h}_{1}(u, v), \hat{h}_{2}(u, v)\right), v\right) \\
\left.\quad=\frac{1+\sqrt{1+4 b}}{2} \hat{h}_{2}(u, v)+(3-\sqrt{1-4 b}) \hat{f}\left(u, v, \hat{h}_{1}(u, v), \hat{h}_{2}(u, v)\right)\right) .
\end{array}\right.
$$

We can obtain the values of $\hat{\alpha}_{i}, \hat{\beta}_{i}, \hat{\gamma}_{i}, i=1,2$, by comparing the coefficients of $u^{2}, u v, v^{2}$ in (12). From the existence theorem for center manifolds, the restrictions of map can be obtained as follows:

$$
u \longmapsto \hat{F}(u, v),
$$


where $\hat{F}(u, v)=-u+2 \sqrt{1+4 b} \hat{f}\left(u, v, \hat{h}_{1}(u, v), \hat{h}_{2}(u, v)\right)$. It is easy to verify that $\hat{F}(0,0)=$ $0, \hat{F}_{u}(0,0)=-1, \hat{F}_{v}(0,0)=0, \hat{F}_{u u}(0,0)=-\frac{2}{2-b}, \hat{F}_{u v}(0,0)=-\frac{1}{b(2-b)}, \hat{F}_{v v}(0,0)=-\frac{1}{2 b^{2}(2-b)}$, $\hat{F}_{\text {uuu }}(0,0)=-\frac{12(1-b)}{b(2-b)^{2}}$, and

$$
\begin{aligned}
& k_{1}=\hat{F}_{u u}(0,0) \hat{F}_{v}(0,0)+2 \hat{F}_{u v}(0,0)=-\frac{2}{b(2-b)}>0, \\
& k_{2}=\frac{1}{2}\left(\hat{F}_{u u}(0,0)\right)^{2}+\frac{1}{3} \hat{F}_{u, u, u}(0,0)=\frac{2}{b(2-b)}<0 .
\end{aligned}
$$

So, map (13) undergoes a flip bifurcation at $(u, v)=(0,0)$, and the periodic- 2 points that bifurcate from $(u, v)=(0,0)$ for $v>0$ are unstable.

As for the case of $b=-\frac{1}{4}$ and $b \in\left(-1,-\frac{1}{4}\right)$, we have the same results by making use of the similar analysis.

Summarizing the above discussion, we have the following results.

Theorem 4 If $b \in(-1,0)$ and $a=a^{*}$, then map (2) undergoes a flip bifurcation at $Z_{2}^{*}$. Moreover, the period-2 points bifurcating from $Z_{2}^{*}$ are unstable for $a>a^{*}$.

\subsection{Naimark-Sacker bifurcation}

In this subsection, we discuss the Naimark-Sacker bifurcation of map (2) occurring at $Z_{2}^{*}$. If $a=a_{h}$ and $b \in(-1,1)$, then the roots of $(6)$ are $\lambda_{1,2}\left(a_{h}\right)=-\frac{b}{2} \pm i \frac{\sqrt{4-b^{2}}}{2}, \lambda_{3}\left(a_{h}\right)=b$. Apparently, $\left|\lambda_{1,2}\left(a_{h}\right)\right|=1,\left|\lambda_{3}\left(a_{h}\right)\right|<1$.

If $a \approx a_{h}$, then the roots of (6) are $\lambda_{1,2}(a)=\alpha \pm i \beta$ and $\lambda_{3}$, where $\left.\alpha\right|_{a=a_{h}}=-\frac{b}{2},\left.\beta\right|_{a=a_{h}}=$ $\frac{\sqrt{4-b^{2}}}{2}$, and $\left.\lambda_{3}\right|_{a=a_{h}}=b$.

Since $\lambda_{1,2}(a)$ satisfy $(6)$, we have that

$$
\left\{\begin{array}{l}
\alpha^{3}-3 \alpha \beta^{3}+\left[(b-1)+\sqrt{4 a+(b-1)^{2}}\right] \alpha-b=0 \\
3 \alpha^{2}-\beta^{2}+\left[(b-1)+\sqrt{4 a+(b-1)^{2}}\right]=0
\end{array}\right.
$$

It is easy to calculate that $\frac{d}{d a}\left|\lambda_{1,2}\left(a_{h}\right)\right|=\frac{1}{(1-b)(2+b)\left(1+2 b^{2}\right)}>0$. Besides, for the NaimarkSacker bifurcation, it requires that $\lambda_{1,2}^{j}\left(a_{h}\right) \neq 1, j=1,2,3,4$, which implies that $b \neq \pm 2$, $b \neq 1, b \neq 0$. So, it requires that $b \neq 0$ since $b \in(-1,1)$.

Let $\bar{x}=x-x_{2}^{*}, \bar{y}=y-y_{2}^{*}, \bar{z}=z-z_{2}^{*}$, then system (2) becomes

$$
\left(\begin{array}{l}
\bar{x} \\
\bar{y} \\
\bar{z}
\end{array}\right) \mapsto\left(\begin{array}{ccc}
0 & -\left(1-b^{2}\right) & b \\
1 & 0 & 0 \\
0 & 1 & 0
\end{array}\right)\left(\begin{array}{l}
\bar{x} \\
\bar{y} \\
\bar{z}
\end{array}\right)+\left(\begin{array}{c}
-\bar{y}^{2} \\
0 \\
0
\end{array}\right) .
$$

Let $(\bar{x}, \bar{y}, \bar{z})^{\prime}=\tilde{T}(u, v, w)^{\prime}$, then map (14) becomes

$$
\left(\begin{array}{l}
u \\
v \\
w
\end{array}\right) \longmapsto\left(\begin{array}{ccc}
-\frac{b}{2} & -\frac{\sqrt{4-b^{2}}}{2} & 0 \\
-\frac{\sqrt{4-b^{2}}}{2} & -\frac{b}{2} & 0 \\
0 & 0 & b
\end{array}\right)\left(\begin{array}{c}
u \\
v \\
w
\end{array}\right)+\tilde{F}(u, v, w)\left(\begin{array}{c}
3 b \\
\sqrt{4-b^{2}} \\
-\sqrt{4-b^{2}}
\end{array}\right)
$$


where

$$
\tilde{T}=\left(\begin{array}{ccc}
-b \frac{\sqrt{4-b^{2}}}{2} & \frac{b^{2}-2}{2} & b^{2} \\
\frac{\sqrt{4-b^{2}}}{2} & -\frac{b}{2} & b \\
0 & 1 & 1
\end{array}\right)
$$

and

$$
\tilde{F}(u, v, w)=\frac{\left(\frac{\sqrt{4-b^{2}}}{2} u-\frac{b}{2} v+b w\right)^{2}}{\left(1+2 b^{2}\right) \sqrt{4-b^{2}}} .
$$

By the similar method as in Sects. 3.1 and 3.2, we can study the stability and the Naimark-Sacker bifurcation of $(\bar{x}, \bar{y}, \bar{z})=(0,0,0)$ near $\bar{a}=0$. Let $W^{c}(0)=\{(u, v, w) \mid w=$ $\tilde{h}(u, v), \tilde{h}(0,0)=0, D \tilde{h}(0,0)=0\}$, where $\tilde{h}(u, v)=\tilde{\alpha} u^{2}+\tilde{\beta} u v+\tilde{\gamma} v^{2}+O(3)$.

Because of invariance of the center manifold [19], we have that

$$
\begin{aligned}
\tilde{h}( & \left.-\frac{b}{2} u-\frac{\sqrt{4-b^{2}}}{2} v+3 b \tilde{F}(u, v, \tilde{h}(u, v)), \frac{\sqrt{4-b^{2}}}{2} u-\frac{b}{2} v+\sqrt{4-b^{2}} \tilde{F}(u, v, \tilde{h}(u, v))\right) \\
& =b w-\sqrt{4-b^{2}} \tilde{F}(u, v, \tilde{h}(u, v)) .
\end{aligned}
$$

We can obtain the values of $\tilde{\alpha}, \tilde{\beta}, \tilde{\gamma}$ by comparing the coefficients of $u^{2}, u v, v^{2}$ in (16). From the existence theorem for center manifolds, the restrictions of map are

$$
\left(\begin{array}{l}
u \\
v
\end{array}\right) \longmapsto\left(\begin{array}{cc}
-\frac{b}{2} & -\frac{\sqrt{4-b^{2}}}{2} \\
\frac{\sqrt{4-b^{2}}}{2} & -\frac{b}{2}
\end{array}\right)\left(\begin{array}{l}
u \\
v
\end{array}\right)+\left(\begin{array}{l}
\tilde{f}(u, v) \\
\tilde{g}(u, v)
\end{array}\right),
$$

where $\tilde{f}(u, v)=3 b \tilde{F}(u, v, \tilde{h}(u, v)), \tilde{g}(u, v)=\sqrt{4-b^{2}} \tilde{F}(u, v, \tilde{h}(u, v))$.

Then we use the method given in [20] to study the Naimark-Sacker bifurcation of map (2). The coefficients are given as follows:

$$
\begin{aligned}
\xi_{20}(u, v) & =\frac{1}{8}\left[\left(\tilde{f}_{u u}-\tilde{f}_{v v}+2 \tilde{g}_{u v}\right)+i\left(\tilde{g}_{u u}-\tilde{g}_{v v}-2 \tilde{f}_{u v}\right)\right] \\
& =\frac{b\left(1-b^{2}+i\left(1+b^{2} \sqrt{4-b^{2}}\right)\right)}{4\left(1+2 b^{2}\right) \sqrt{4-b^{2}}}, \\
\xi_{11}(u, v) & =\frac{1}{4}\left[\left(\tilde{f}_{u u}+\tilde{f}_{v v}\right)+i\left(\tilde{g}_{u u}+\tilde{g}_{v v}\right)\right] \\
& =\frac{3 b+i \sqrt{\left(4-b^{2}\right)}}{2\left(1+2 b^{2}\right) \sqrt{4-b^{2}}}, \\
\xi_{02}(u, v) & =\frac{1}{8}\left[\left(\tilde{f}_{u u}-\tilde{f}_{v v}-2 \tilde{g}_{u v}\right)+i\left(\tilde{g}_{u u}-\tilde{g}_{v v}+2 \tilde{f}_{u v}\right)\right] \\
& =\frac{b\left(5-2 b^{2}\right)+i\left(1-2 b^{2}\right) \sqrt{4-b^{2}}}{4\left(1+2 b^{2}\right) \sqrt{4-b^{2}}}, \\
\xi_{21}(u, v) & =\frac{1}{16}\left[\left(\tilde{f}_{u u u}+\tilde{f}_{u v v}+\tilde{g}_{u u v}+\tilde{g}_{v v v}\right)+i\left(\tilde{g}_{u u u}+\tilde{g}_{u v v}-\tilde{f}_{u u v}-\tilde{f}_{v v v}\right)\right] \\
& =\left[b \left(-2 i-8 b i-10 b^{2} i+3 b^{3} i-3 b^{4} i+2 b^{5} i-5 b^{2} \sqrt{4-b^{2}}-3 b^{3} \sqrt{4-b^{2}}\right.\right.
\end{aligned}
$$




$$
\begin{aligned}
& \left.\left.\quad+2 b^{4} \sqrt{4-b^{2}}\right)\right] \cdot\left[4(1-b) \sqrt{4-b^{2}}\left(1+2 b^{2}\right)^{2}\left(1+2 b+b^{2}-b^{3}\right)\right]^{-1}, \\
& k=-\operatorname{Re}\left[\frac{(1-2 \lambda)(\bar{\lambda})^{2}}{1-\lambda} \xi_{11} \xi_{20}\right]-\frac{1}{2}\left|\xi_{11}\right|^{2}-\left|\xi_{02}\right|^{2}+\operatorname{Re}\left[\bar{\lambda} \xi_{21}\right] \\
& =\frac{(1+b)\left(2 b^{2}-2 b-3\right)}{4\left(1-b(2+b)\left(1+2 b^{2}\right)\left(1+2 b+b^{2}-b^{3}\right)\right.} .
\end{aligned}
$$

We can easily see that $k>0$ for $b \in\left(-1,-\frac{\sqrt{7}-1}{2}\right)$ and $k<0$ for $b \in\left(-\frac{\sqrt{7}-1}{2}, 1\right)$. From the above analysis, we have the following results.

Theorem 5 Assume that $b \in(-1,1)$ and $b \neq 0$. If $b \in\left(-1,-\frac{\sqrt{7}-1}{2}\right)$, map (2) undergoes a subcritical Naimark-Sacker bifurcation at $Z_{2}^{*}$ for $a=a_{h}$, and a repelling invariant circle occurs for $a<a_{h} ;$ if $b \in\left(-\frac{\sqrt{7}-1}{2}, 1\right)$, the map undergoes a supercritical Naimark-Sacker bifurcation at $Z_{2}^{*}$ for $a=a_{h}$, and an attracting invariant circle occurs for $a>a_{h}$.

\section{Numerical simulations}

In this section, rich complex dynamical behaviors of system (2) are displayed by the bifurcation graphs, maximum Lyapunov exponents, and phase portraits. The numerical simulations also support theoretical analysis.

\subsection{Numerical simulations for stability and codimension one bifurcations of fixed points}

In this subsection, some numerical simulations verify the correctness of Proposition 2 and Theorems 3-5. Four cases are considered as follows:

Case(1) Let $b=0.5$, then $a_{0}=-0.0625, a^{*}=0.1875$, and $a_{h}=0.3281$. From Proposition 2, the fixed point $Z_{2}^{*}$ of system (2) is stable for $a \in\left(a_{0}, a_{h}\right)$. System (2) undergoes a fold bifurcation at $Z_{0}^{*}$ for $a=a_{0}$ by Theorem 3 . Since $b \in\left(-\frac{\sqrt{7}-1}{2}, 1\right)$, we have that the super Naimark-Sacker bifurcation occurs at $Z_{2}^{*}$ for $a=a_{h}$ by Theorem 5. Figure 2(a) shows the correctness of Proposition 2 and Theorems 3-5.

Case (2) Let $b=-0.5$, then $a_{0}=-0.5625, a^{*}=0.3125$, and $a_{h}=0.7031$. From Proposition 2 , we have that the fixed point of system (2) $Z_{2}^{*}$ is stable for $a \in\left(a^{*}, a_{h}\right)$. Map (2) undergoes a fold bifurcation at $Z_{0}^{*}$ for $a=a_{0}$ by Theorem 3 . Since $k_{2}<0$, we have that the unstable flip bifurcation occurs at $Z_{2}^{*}$ for $a=a^{*}$ by Theorem 4 . Since $b \in\left(-\frac{\sqrt{7}-1}{2}, 1\right)$, we have that the super Naimark-Sacker bifurcation occurs at $Z_{2}^{*}$ for $a=a_{h}$ by Theorem 5 . Figure 2(b) shows the correctness of Proposition 2 and Theorems 3-5.

Case (3) Let $b=-0.85, a_{0}=-0.8556, a^{*}=-0.1331$, and $a_{h}=0.2759$. From Proposition 2, we have that the fixed point $Z_{2}^{*}$ is stable for $a \in\left(a^{*}, a_{h}\right)$. Map (2) undergoes a fold bifurcation at $Z_{0}^{*}$ for $a=a_{0}$ by Theorem 3. Since $k_{2}<0$, we have that the unstable flip bifurcation occurs at $Z_{2}^{*}$ for $a=a^{*}$ by Theorem 4 . Since $b \in\left(-1,-\frac{\sqrt{7}-1}{2}\right)$, we have that the subcritical Naimark-Sacker bifurcation occurs at $Z_{2}^{*}$ for $a=a_{h}$ by Theorem 5. Figure 2(c) shows the correctness of Proposition 2 and Theorems 3-5.

Case (4) Fig. 2(d) shows the local bifurcation diagram for $b=-0.85$ and $a \in(0.273,0.279)$. For $b=-0.85, a_{h}=0.2759$, Theorem 5 tells us that map (2) undergoes a subcritical Naimark-Sacker bifurcation at $Z_{2}^{*}$ for $a=a_{h}$ and has a small amplitude unstable invariant cycle for $a<a_{h}$ slightly. However, Fig. 2(d) exhibits that there exists a large amplitude stable invariant cycle in map (2) for $a \in(0.274,0.279)$. Note that $a_{h} \in(0.274,0.279)$. So, we infer that there exist a stable invariant cycle and an unstable one at the same time for 


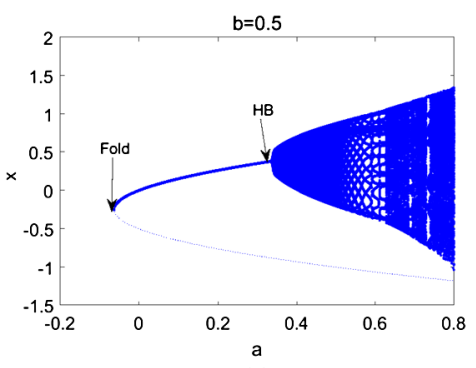

(a)

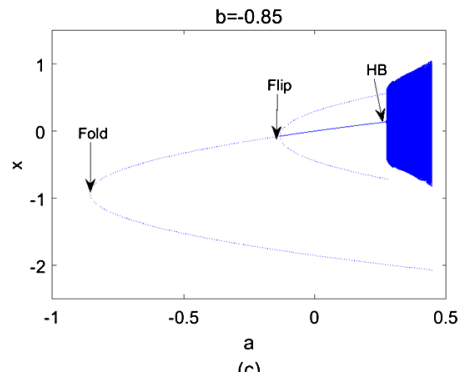

(c)
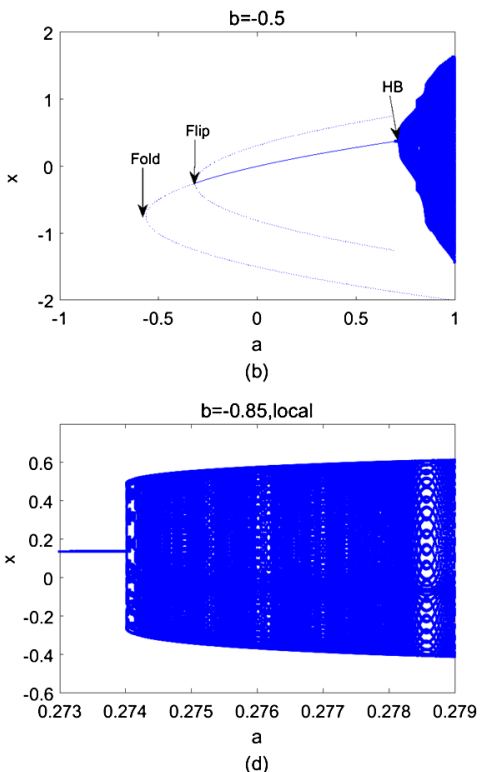

(d)

Figure 2 (a)-(c) show bifurcation diagrams of system (2) in the $(a, x)$ plane for $b=0.5, b=-0.5$, and $b=-0.85$, respectively. Here, the fold bifurcation, flip bifurcation, and Naimark-Sacker bifurcation are labeled as "Fold", "Flip", and "HB", respectively. (d) shows the local enlarged diagram of (c) for $a \in(0.273,0.279)$

$a \in\left(0.274, a_{h}\right)$ in map (2). Furthermore, the stable invariant cycle is outside the unstable invariant cycle and they coincide with each other to be a semi-stable invariant cycle at $a=0.274$. So the system has a double-cycle structure for $a \in\left(0.274, a_{h}\right)$. Because the small amplitude invariant cycle is unstable, it cannot be plotted in the figure. But the large amplitude stable invariant cycle can be observed from Fig. 2(d), Fig. 7(a), and Fig. 8(a).

\subsection{Further numerical simulations for system (2)}

In this subsection, some numerical simulations show that system (2) has some new dynamical behaviors as its parameters vary.

Next, we give numerical simulations in three cases for $b=0.5, b=-0.5$, and $b=-0.85$, respectively.

Case (A) Let $b=0.5$, then $a_{h}=0.3281$. We illustrate the bifurcation diagram and the maximum Lyapunov exponent of system (2) for $a \in(0.3,0.8)$ in Figs. 3(a) and 3(b), respectively. In Fig. 3(a), the fixed point is stable for $a \in\left(0.3, a_{h}\right)$. With the increase of the parameter $a$, the stable fixed point will become unstable. The Naimark-Sacker bifurcation occurs at $a \sim a_{h}$. Simultaneously, a stable invariant circle will appear as $a$ is slightly greater than $a_{h}$. Figure 3(c) is the local enlarged diagram of Fig. 3(a) for $a \in(0.73,0.732)$, which shows the process from the period- 10 orbits at $a=0.73085$ to the chaotic region. We can also observe several periodic windows in the chaotic regions. In Fig. 3(b), the maximal Lyapunov exponent can rapidly change its values between negative and positive numbers. We deduce that there are stable fixed points or stable periodic windows in the chaotic region when the maximal Lyapunov exponent is negative. Fig. 4 shows phase portraits for $a=a_{h}+0.02, a=a_{h}+0.15, a=0.73085$, and $a=a_{h}+0.4$, which correspond to stable invariant cycle, stable quasi-invariant cycle, periodic-10 orbit, and chaos, respectively.

Case (B) Let $b=-0.5$, then $a_{h}=0.7031$. We illustrate the bifurcation diagram and the maximum Lyapunov exponent of system (2) for $a \in(0.7,1.0)$ in Figs. 5(a) and 5(b), respec- 

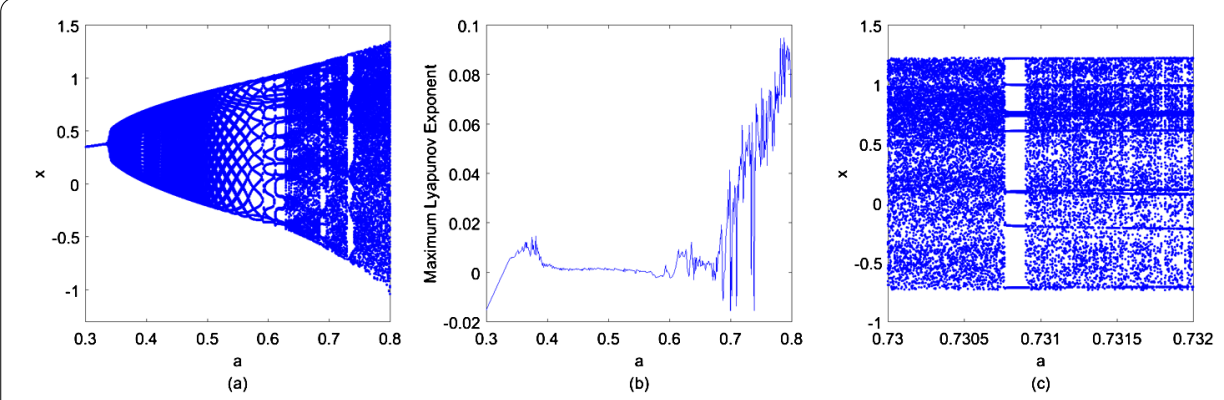

Figure 3 (a) Bifurcation diagram of fixed points of system (2) in the $(a, x)$ plane for $a \in(0.3,0.8)$ and $b=0.5$; (b) Maximum Lyapunov exponent corresponding to (a); (c) The local amplified bifurcation diagram of (a) for $a \in(0.73,0.732)$

Figure 4 Phase portraits for $b=0.5$ :

(a) $a=a_{h}+0.02$; (b) $a=a_{h}+0.15$; (c) $a=0.73085$;

(d) $a=a_{h}+0.40$, where, $a_{h}=0.3281$
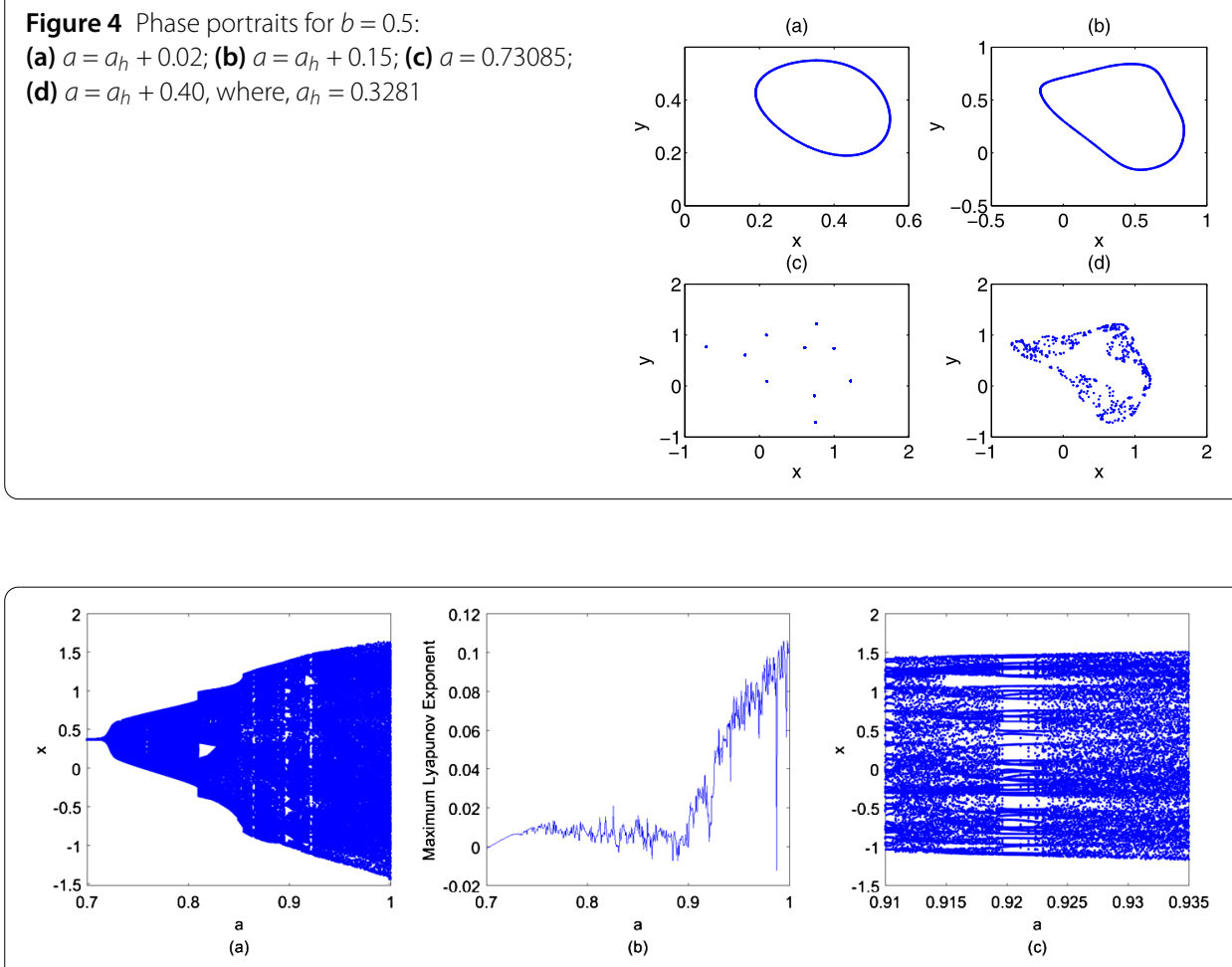

Figure 5 (a) Bifurcation diagram of fixed points of system (2) in the $(a, x)$ plane for $a \in(0.7,1.0)$ and $b=-0.5$; (b) Maximum Lyapunov exponent corresponding to (a); (c) The local amplified bifurcation diagram of (a) for $a \in(0.91,0.935)$

tively. Figure 5(c) is the local enlarged diagram of Fig. 5(a) for $a \in(0.91,0.935)$. As $a$ varies, we can see the existence of chaotic regions and periodic windows. From Fig. 5(b), the maximal Lyapunov exponent can rapidly change its values between negative and positive as $a \in(0.85,1.0)$. We deduce that there are stable fixed points or stable periodic windows in the chaotic region when the maximal Lyapunov exponent is negative. From Fig. 5(c) we observed that there is a periodic window at $a \sim 0.92205$. Fig. 6 shows phase portraits for $a=a_{h}+0.02, a=a_{h}+0.1, a=0.92205$, and $a=a_{h}+0.15$, which correspond to stable invariant cycle, stable quasi-invariant cycle, periodic orbit, and chaos, respectively. 
Figure 6 Phase portraits for $b=-0.5$ :

(a) $a=a_{h}+0.02 ;$ (b) $a=a_{h}+0.1 ;$ (c) $a=0.92205$;

(d) $a=a_{h}+0.15$, where $a_{h}=0.7031$
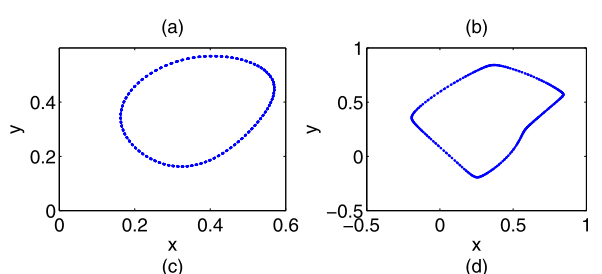

(c)
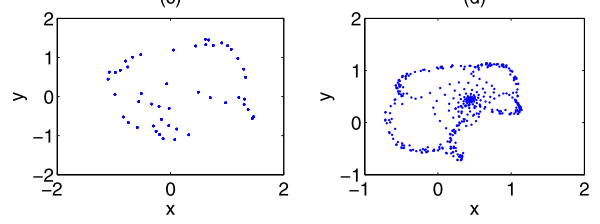
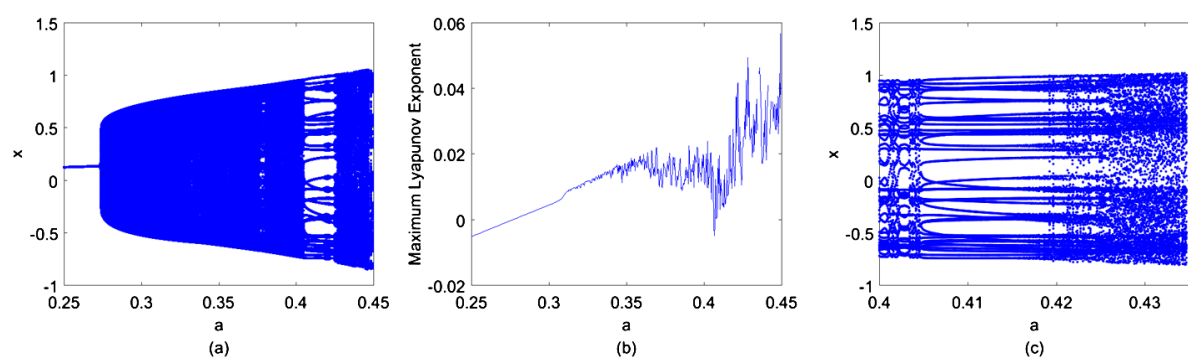

Figure 7 (a) Bifurcation diagram of fixed points of system (2) in the $(a, x)$ plane for $a \in(0.25,0.45)$ and $b=-0.85$; (b) Maximum Lyapunov exponent corresponding to (a); (c) The local amplified bifurcation diagram of (a) for $a \in(0.4,0.435)$

Figure 8 Phase portraits for $b=-0.85$ :

(a) $a=a_{h}-0.001$; (b) $a=a_{h}+0.02$; (c) $a=0.415$;

(d) $a=a_{h}+0.15$, where $a_{h}=0.2759$
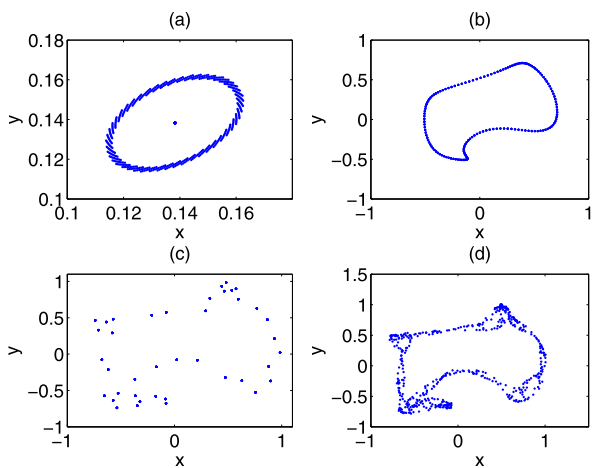

Case (C) Let $b=-0.85$, then $a_{h}=0.2759$. We illustrate the bifurcation diagram and the maximum Lyapunov exponent of system (2) for $a \in(0.25,0.45)$ in Figs. $7(\mathrm{a})$ and $7(\mathrm{~b})$, respectively. Figure 7(c) is the local enlarged diagram of Fig. 7(a) for $a \in(0.4,0.435)$. With the increase of the parameter $a$, we can see the existence of chaotic regions and periodic windows. Similar to the case in Fig. 5 (b), we can see as $a \in(0.35,0.45)$, some Lyapunov exponents are positive and some are negative in Fig. 7(b). We can also expect that there are stable fixed points or stable periodic windows in the chaotic region as the maximal Lyapunov exponent is negative. Figure 7(c) shows the process from approximate period-40 orbits to chaos at $a \sim 0.415$. Fig. 8 shows phase portraits for $a=a_{h}-0.001, a=a_{h}+0.02, a=0.415$, and $a=a_{h}+0.15$, which correspond to stable invariant cycle, stable quasi-invariant cycle, periodic orbit, and chaos, respectively. 


\section{Conclusion}

In this paper we use the analytic and numerical methods to study the three-dimensional Hénon map with non-constant Jacobian determinant. From the bifurcation theory and center manifold theorem, we know that system (2) can undergo the fold bifurcation, flip bifurcation, and Naimark-Sacker bifurcation. The analytic results are illustrated by numerical simulations. We observe a number of interesting phenomena, such as the process of bifurcation to chaos, period windows, invariant cycle, coexistence of periodic orbit and chaos, etc., from bifurcation diagrams, maximum Lyapunov exponents, and phase portraits. In particular, we find coexistence of an unstable invariant cycle and a stable invariant cycle in the system for $b=-0.85$ from bifurcation diagrams and phase portraits. Due to the specific form of high-dimensional generalized Hénon map, we believe that some conclusions in the paper can be extended to high-dimensional generalized Hénon map easily. The research methods and results of our paper will contribute to the study of the generalized Hénon map.

\section{Acknowledgements}

The authors would like to thank the referee and the editor for their valuable comments which led to improvement of this work. This work was supported by the National Natural Science Foundation of China (Nos. 10971009, 10771196, 11771033), the National Scholarship Fund (No. 201303070222, 201706020203).

\section{Competing interests}

The authors declare that they have no competing interests.

\section{Authors' contributions}

All authors participated in drafting and checking the manuscript, read and approved the final manuscript.

\section{Author details}

${ }^{1}$ School of Mathematics and Systems Science, Beihang University, Beijing, P.R. China. ${ }^{2}$ School of Geographical and Earth Sciences, University of Glasgow, Glasgow, United Kingdom.

\section{Publisher's Note}

Springer Nature remains neutral with regard to jurisdictional claims in published maps and institutional affiliations.

Received: 4 January 2018 Accepted: 26 April 2018 Published online: 16 May 2018

\section{References}

1. Hénon, M.: A two-dimensional mapping with a strange attractor. Commun. Math. Phys. 50, 69-77 (1976)

2. Arai, Z: On loops in the hyperbolic locus of the complex Hénon map and their monodromies. Physica D 334 133-140 (2016)

3. Bedford, E., Smillie, J.: A symbolic characterization of the horseshoe locus in the Hénon family. Ergod. Theory Dyn. Syst. 37, 1389-1412 (2017)

4. Paramio, M., Sesma, J.: Invariant directions in the Hénon map. Phys. Lett. A 132, 98-100 (1988)

5. Shi, Y.G.: A note on homoclinic or heteroclinic orbits for the generalized Hénon map. Acta Math. Appl. Sin. Engl. Ser. 32, 283-288 (2016)

6. Ping, P., Xu, F., Mao, Y., Wang, Z:: Designing permutation-substitution image encryption networks with Henon map. Neurocomputing 283, 53-63 (2018)

7. Alligood, K.T., Sauer, T.: Rotation numbers of periodic orbits in the Hénon map. Commun. Math. Phys. 120, 105-119 (1988)

8. Lorenz, E.N.: Compound windows of the Hénon map. Physica D 237, 1689-1704 (2008)

9. Shukla, M., Sharma, B.: Investigation of chaos in fractional order generalized hyperchaotic Henon map. AEÜ, Int. J. Electron. Commun. 78, 265-273 (2017)

10. Baier, G., Klein, M.: Maximum hyperchaos in generalized Hénon maps. Phys. Lett. A 151, $281-284$ (1990)

11. Dullin, H.R., Meiss, J.D.: Generalized Hénon maps: the cubic diffeomorphisms of the plane. Physica D 143, 262-289 (2000)

12. Richter, H.: The generalized hénons: examples for higher dimensional chaos. Int. J. Bifurc. Chaos Appl. Sci. Eng. 12, 1371-1384 (2002)

13. An, H.L., Chen, Y.: The function cascade synchronization scheme for discrete-time hyperchaotic systems. Commun. Nonlinear Sci. Numer. Simul. 14, 1494-1501 (2009)

14. Filali, R.L., Hammami, S., Benrejeb, M., Borne, P.: On synchronization, anti-synchronization and hybrid synchronization of 3D discrete generalized Hénon map. Nonlinear Dyn. Syst. Theory 12, 81-95 (2012)

15. Senti, S., Takahasi, H.: Equilibrium measures at temperature zero for Hénon-like maps at the first bifurcation. SIAM J. Appl. Dyn. Syst. 15, 106-124 (2016) 
16. Hitzl, D.L., Zele, F.: An exploration of the Hénon quadratic map. Physica D 14, 305-326 (1985)

17. Gonchenko, S.V., Ovsyannikov, I.I., Simó, C., Turaev, D.: Three-dimensional Hénon-like maps and wild Lorenz-like attractors. Int. J. Bifurc. Chaos Appl. Sci. Eng. 15, 3493-3508 (2005)

18. Gonchenko, A.S., Gonchenko, S.V.: Variety of strange pseudohyperbolic attractors in three-dimensional generalized Hénon maps. Physica D 337, 43-57 (2016)

19. Winggins, S.: Introduction to Applied Nonlinear Dynamical Systems and Chaos. Texts in Applied Mathematics, vol. 2. Springer, New York (1990)

20. Guckenheimer, J., Holmes, P.: Nonlinear Oscillations, Dynamical Systems, and Bifurcations of Vector Fields. Applied Mathematical Sciences., vol. 42. Springer, New York (1990)

Submit your manuscript to a SpringerOpen ${ }^{\circ}$ journal and benefit from:

- Convenient online submission

- Rigorous peer review

- Open access: articles freely available online

- High visibility within the field

- Retaining the copyright to your article

Submit your next manuscript at $\gg$ springeropen.com 